\title{
1-Methyl-4-phenyl-1,2,3,6-tetrahydropyridine induced Parkinson's disease in zebrafish
}

Nukala Sarath Babu*\#, ChLN. Murthy, S. Kakara, R. Sharma, CVB Swamy and Mohammed M. Idris* ${ }^{*} \mathrm{CSIR}$ - Centre for Cellular and Molecular Biology (CCMB), Hyderabad, India \# Department of Pharmaceutical Sciences, University of Milan, Milan, Italy.

UNIVERSITÀ DEGLI STUDI DI MILANO

Parkinson's disease (PD) is the most common age associated neurodegenerative disease, which has been extensively studied for its etiology and phenotype. PD has been widely studied in alternate model system such as rodents towards understanding the role of neurotoxin by inducing PD. This study is aimed to understand the biomechanism of PD inducing PD. This study is aimed to understand the biomechanism of PD in zebrafish model system induced by 1 -methyl-4-phenyl-1,2,3,6tetrahydropyridine (MPTP). The phenotype and role of various genes and proteins for Parkinsonism were tested and evaluated in this study using behaviour, molecular and proteomic approaches. Zebrafish PD model induced by 1-methyl- 4-phenyl-1,2,3,6-tetrahydropyridine showed a significant level of decrease in the movement with erratic swimming pattern and increased freezing bouts. CHCHD2, EEF2B, LRRK2, PARK7, PARK2, POLG, SNCGB and SYNB genes were differentially regulated at the transcript level in PD zebrafish. Similarly a total of 73 proteins were recognized as differentially expressed in the nervous system of zebrafish due to Parkinsonism based on quantitative proteomics approach. Proteins such as NEFL,MUNC13-1, NAV2 and GAPVD1 were down regulated in the zebrafish brain for the PD phenotype, which were associated with the neurological pathways. This zebrafish based PD model can be used as a potential model system for screening prospective drug molecules for $\mathrm{PD}$.

\section{Objective}

To establish the zebrafish as a model organism for studying the Parkinson's Disease.

system involving behavioural, transcriptomics and proteomics studies.



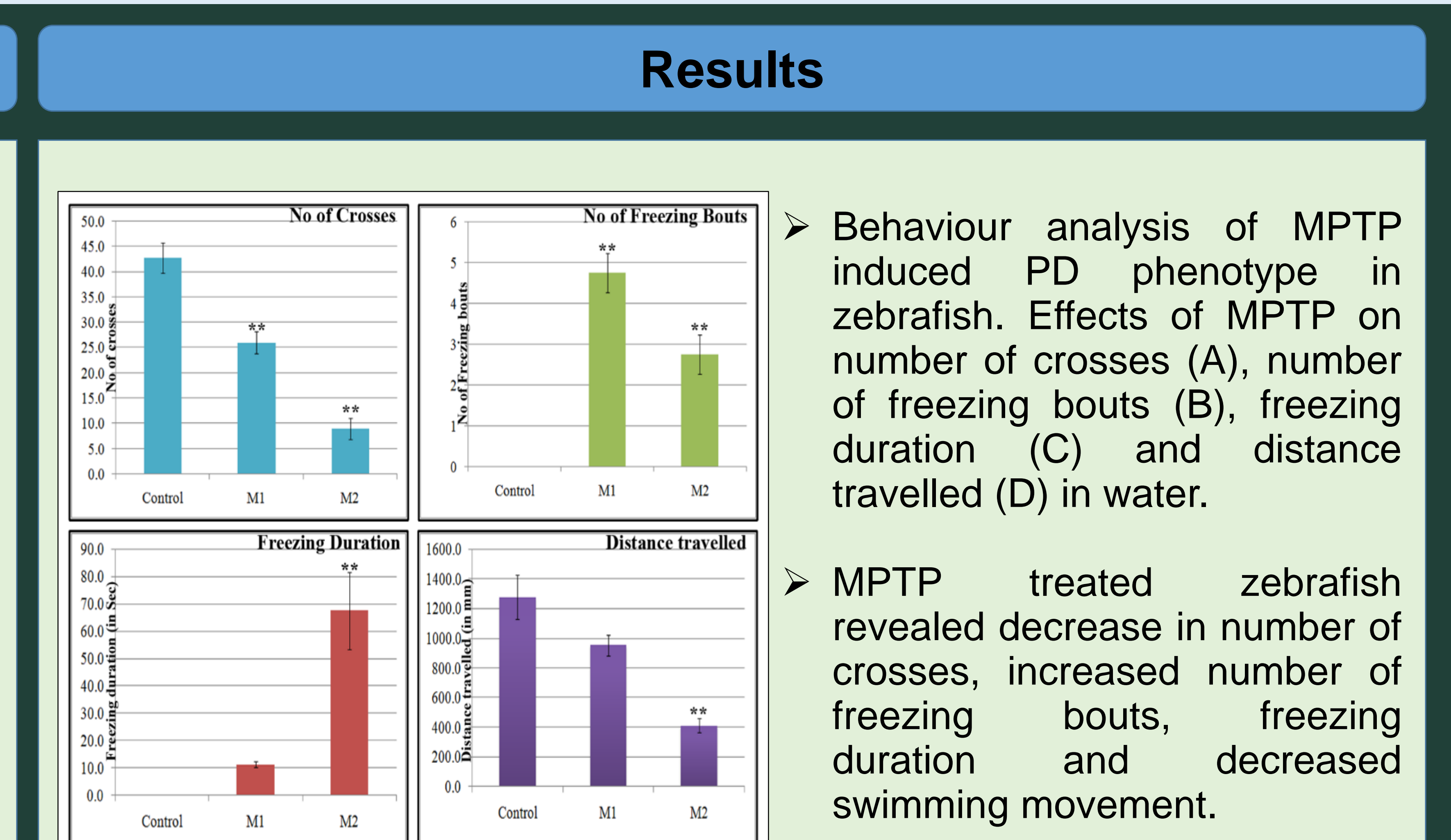

Color Key

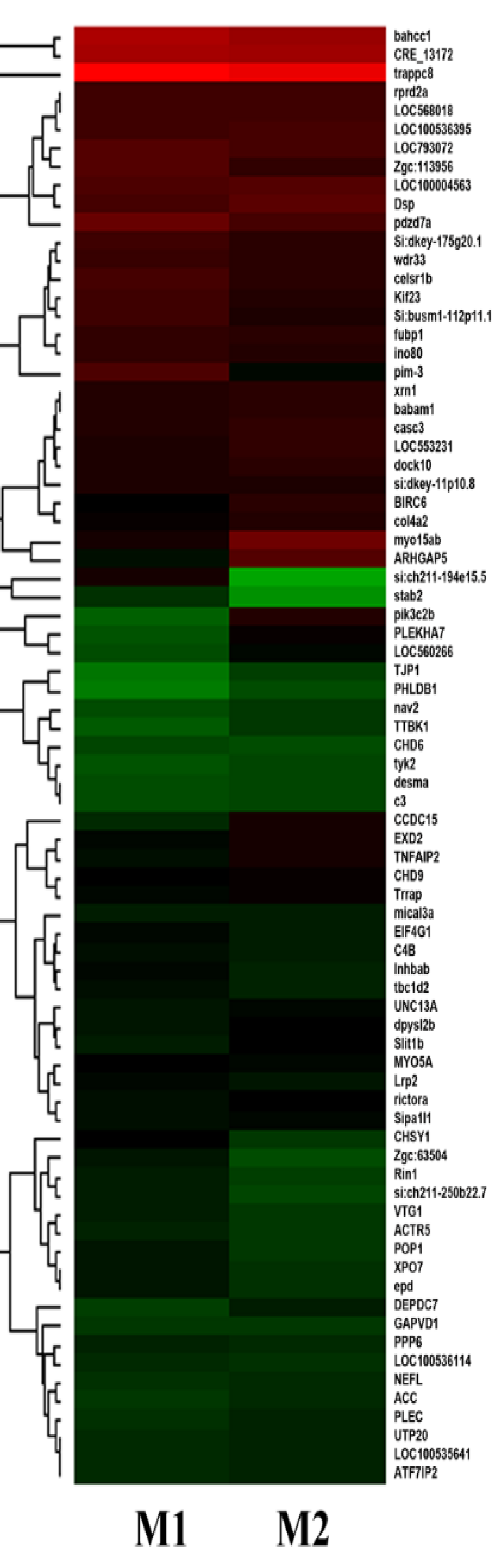

- Heat map view of protein zebrafish in PD induced zebrafish brain. The protein expressions were calculated protein 2 log fold changes in Prote expression based control and 1-dose MPTP treatment and 2-dose of MPTP treatment. The hierarchical cluster heat map expression was calculated using $R$ program.

Proteins such as NEFL and Plectin 1 were found down regulated in zebrafish brain, which were found to be involved in cytoskeleton remodelling of neurofilaments, the major intermediate filaments of the neurons.

The cyclin-dependent kinase (CDK5), which has a major role in neuronal development and differentiation, was also found associated with PD in zebrafish by the down regulation of NEF
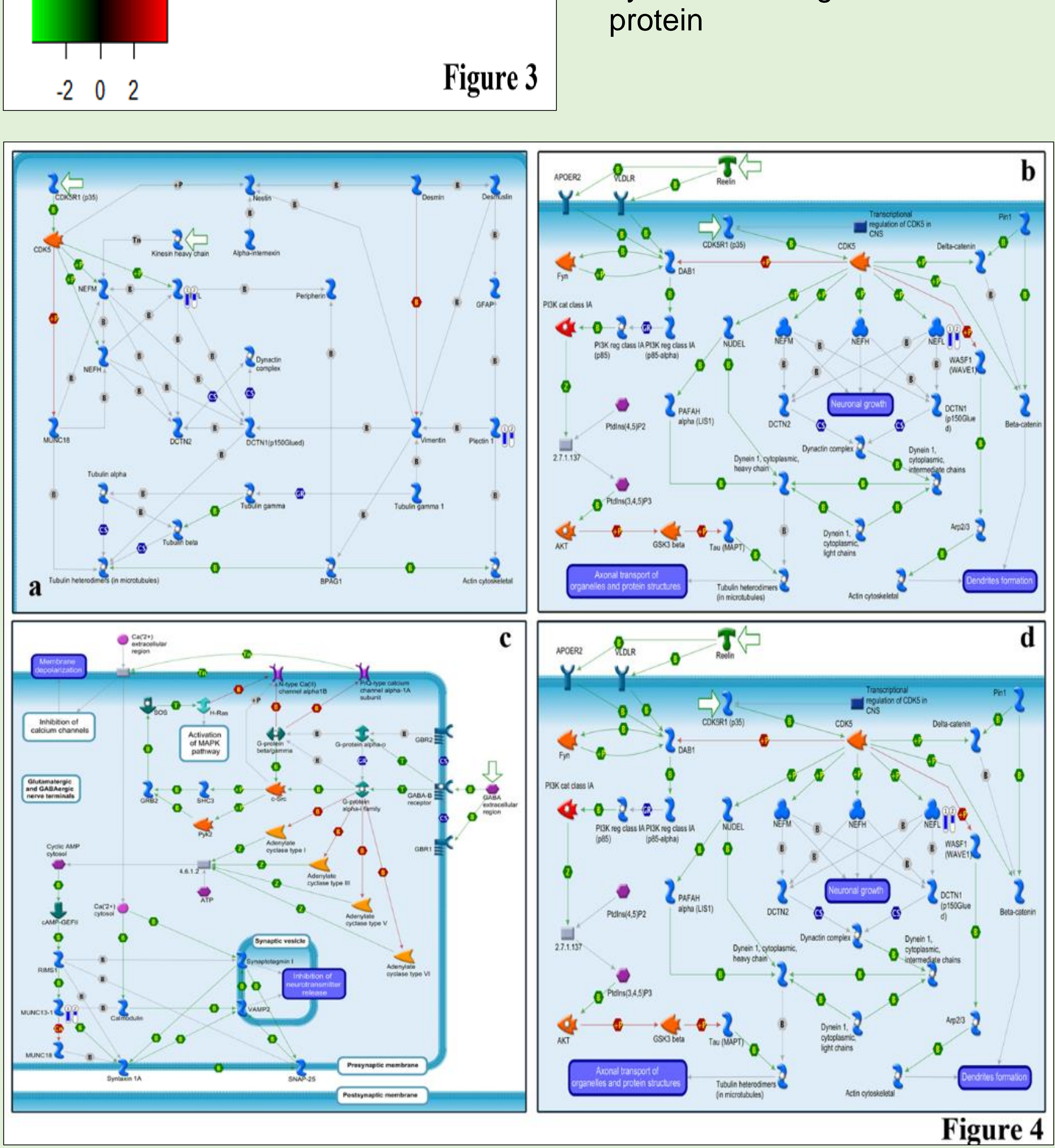

Network pathway map analysis obtained from the differentially expressed proteome of PD zebrafish brain. (A) Cytoskeleton remodelling of neurofilaments, (B) role of CDK5 in neuronal development, (C) neurophysiological synaptic vesicle fusion and (D) GABA-B receptor
signalling in presynaptic nerve terminals pathway. The blue colour signaling in presynaptic nerve terminals pathway. The blue colour
thermometer indicates the down-regulation of the proteins identified in the study.

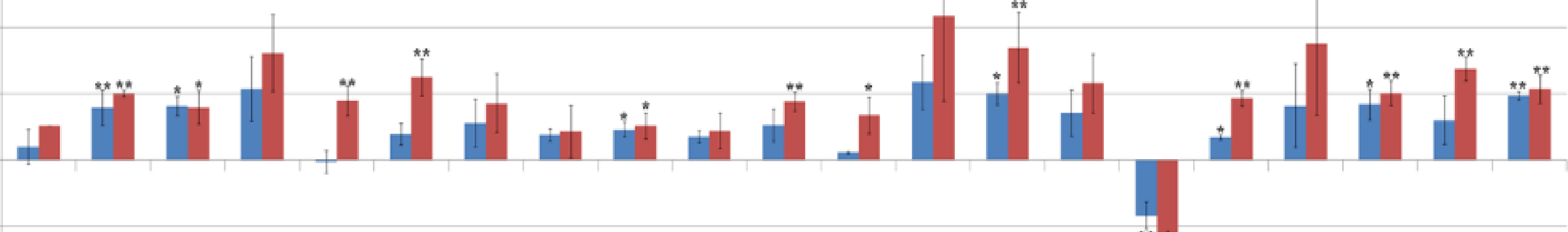

Relative expression of PD genes based on quantitative RT-PCR analysis The relative expression of genes was shown for M1 and M2 dose of MPTP
in zebrafish brain against the control expression. The gene expression in zebrafish brain against the control expression. The gene expression group, ${ }^{* \star} p<0.01$ vs. control group, (one-way ANOVA post test, $n=3$ ).

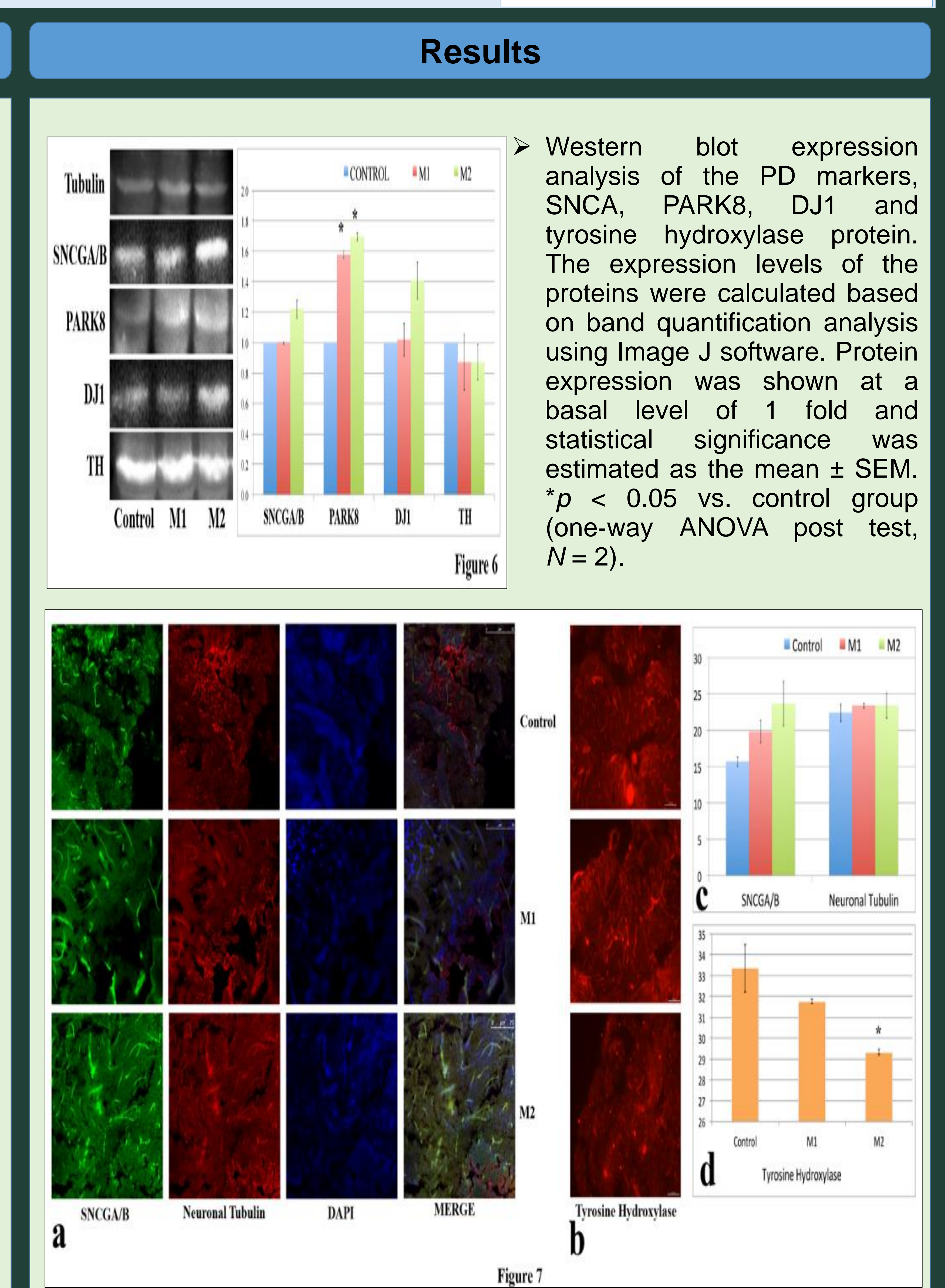

Immunofluorescence analysis of the SNCA protein expression in the cross section of the zebrafish optic tectum. (A) Expression pattern of SNCA, neuronal tubulin, DAPI staining and Merge image, (B) expression patter of tyrosine hydroxylase, (C) relative expression of SNCGA/B protein and $(D)$ relative expression of tyrosine hydroxylase. The analyses were performed on control; M1 and M2 MPTP treated optic tectum sections. The expression was shown as relative expression against control with mean \pm SEM. ${ }^{*} p<0.05$ vs. control group (one-way ANOVA post test, $N=3$ ).

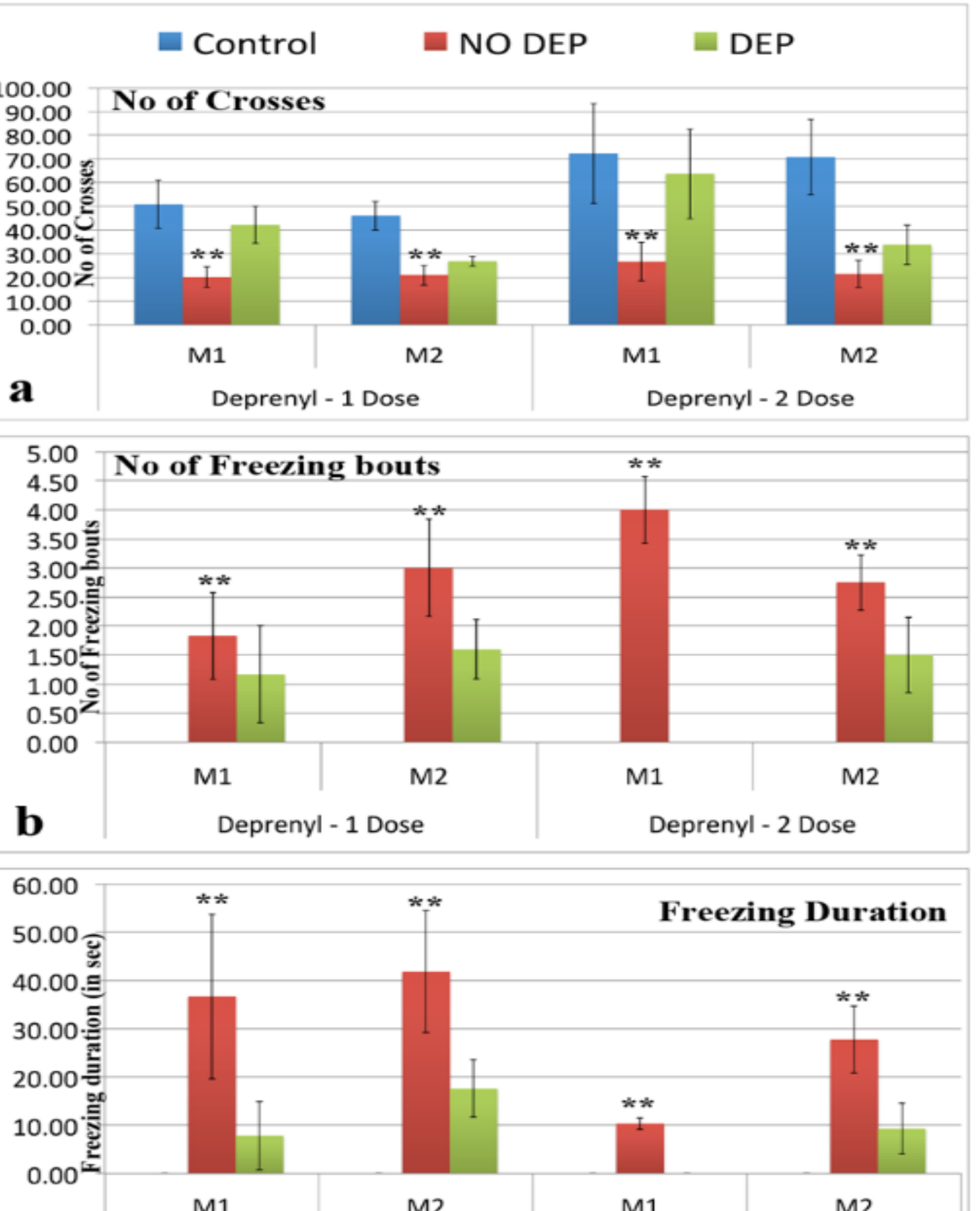

Behaviour analysis of depreny treated PD zebrafish. (A) Effects of MPTP and deprenyl treatment for number of crosses, (B) number of freezing bouts and (C) freezing duration of PD zebrafish.

Numbers of crosses were found significantly recovered in $\mathrm{M} 1$ group for both 1 and 2-dose of deprenyl. Similarly 2-dose deprenyl showed significant bouts and freezing duration in $\mathrm{M} 1$ PD. The recovery of PD phenotype in M2 was found mildly and non-significantly retrieved upon deprenyl treatment.

Significance of the study

The phenotype and role of various genes and proteins involved in Parkinsonism was identified in the MPTP induced zebrafish PD mode based on behavioural molecular and proteomics approaches. $\mathrm{CHCHD2}$ EEF2B, LRRK2 PARK7, PARK2, POLG, SNCGB and SYNB were found associated with Parkinsonism in zebrafish PD model. The model can be used as a potential neurological model for PD study.

\section{Concluding remarks}

This study through quantitative proteomic analysis has established association of 78 proteins as differentially regulated in the zebrafish brain induced with MPTP for PD phenotype.

mRNA expression analysis confirms the Parkinsonism in the MPTP treated zebrafish with up regulation of a group of molecular markers such as CHCHD2, CRF, DNM2A, EIF4G1, SNCGA, SNCGB, SNCB, etc

The model of PD in zebrafish has not only established an alternate model for the PD but a potential model for screening drug targets for an

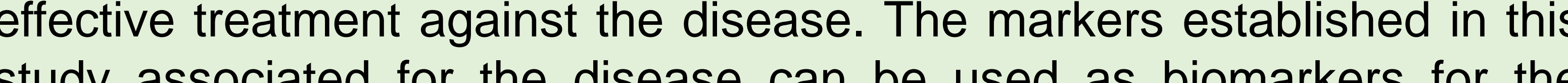
disease and as screening targets for potential PD drugs.

This work was supported and funded by Council for Scientific and Industrial Research (CSIR) network project miND (BSC0115) and InDepth (BSC0111), India.

Publication

Sarath Babu, Nukala, et al. "1-Methyl-4-phenyl-1, 2, 3, 6-tetrahydropyridine induced Parkinson's disease in zebrafish " Proteomics 16.9 (2016): 1407- 